\title{
Osteoblast differentiation and migration are regulated by Dynamin GTPase activity
}

\author{
Pierre P. Eleniste, \\ Indiana University School of Dentistry, Department of Oral Biology, Indianapolis, Indiana, USA \\ Su Huang, \\ Indiana University School of Dentistry, Department of Oral Biology, Indianapolis, Indiana, USA \\ Kornchanok Wayakanon, \\ Indiana University School of Dentistry, Department of Oral Biology, Indianapolis, Indiana, USA \\ Heather W. Largura, and \\ Indiana University School of Dentistry, Department of Oral Biology, Indianapolis, Indiana, USA \\ Angela Bruzzaniti \\ Indiana University School of Dentistry, Department of Oral Biology, Indianapolis, Indiana, USA
}

Pierre P. Eleniste: pelenist@iupui.edu; Su Huang: suhuang63@gmail.com; Kornchanok Wayakanon: kornwaya@iupui.edu; Heather W. Largura: hlargura@iupui.edu; Angela Bruzzaniti: abruzzan@iu.edu

\section{Abstract}

Bone formation is controlled by osteoblasts but the signaling proteins that control osteoblast differentiation and function are still unclear. We examined if the dynamin GTPase, which is associated with actin remodeling and migration in other cells, plays a role in osteoblast differentiation and migration. Dynamin mRNA was expressed in primary osteoblasts throughout differentiation (0-21 days). However, alkaline phosphatase (ALP) activity, a marker of osteoblast differentiation, was decreased in osteoblasts over-expressing dynamin. Conversely, ALP activity was increased following shRNA-mediated knockdown of dynamin and in osteoblasts treated with the dynamin inhibitor, dynasore. Dynasore also reduced c-fos and osterix expression, markers of early osteoblasts, suggesting a role for dynamin in pre-osteoblast to osteoblast differentiation. Since dynamin GTPase activity is regulated by tyrosine phosphorylation, we examined the mechanism of dynamin dephosphorylation in osteoblasts. Dynamin formed a protein complex with the tyrosine phosphatase PTP-PEST and inhibition of phosphatase activity increased the level of phosphorylated dynamin. Further, PTP-PEST blocked the Src-mediated increase in the phosphorylation and GTPase activity of wild-type dynamin but not the phosphorylation mutant dynY231F/Y597F. Although ALP activity was increased in osteoblasts expressing GTPasedefective dynK44A, and to a lesser extent dynY231F/Y597F, osteoblast migration was significantly inhibited by dynK44A and dynY231F/Y597F. These studies demonstrate a novel role for dynamin GTPase activity and phosphorylation in osteoblast differentiation and migration, which may be important for bone formation.

(C) 2013 Elsevier Ltd. All rights reserved.

Correspondence should be addressed to Dr. Angela Bruzzaniti, Indiana University School of Dentistry, Department of Oral Biology DS241, 1121 W. Michigan Street, Indianapolis, 46202, IN, USA, 317-278-3742 (tel), 317-278-1411 (fax), abruzzan@iu.edu.

Conflict of Interest

None declared.

Publisher's Disclaimer: This is a PDF file of an unedited manuscript that has been accepted for publication. As a service to our customers we are providing this early version of the manuscript. The manuscript will undergo copyediting, typesetting, and review of the resulting proof before it is published in its final citable form. Please note that during the production process errors may be discovered which could affect the content, and all legal disclaimers that apply to the journal pertain. 


\section{Keywords}

dephosphorylation; PTP-PEST; alkaline phosphatase; tyrosine phosphorylation

\section{Introduction}

Bone mass is regulated by the activity of osteoclasts which degrade bone and osteoblasts which form new bone (Tanaka et al., 2005). Bone formation at eroded bone surfaces begins by the recruitment of osteoblast precursors which undergo differentiation and maturation into osteoblasts capable of secreting collagen which is later mineralized to form bone (Ducy et al., 2000, Mackie, 2003). Although several key proteins involved in osteoblast function have been identified, much remains to be understood about the process of osteoblast recruitment, migration and differentiation into mature bone-forming cells.

Dynamin is a GTP hydrolase (GTPase) that participates in several cellular activities such as endocytosis, intracellular membrane trafficking (Hinshaw, 2000, Oh et al., 1998, Schmid et al., 1998, Song and Schmid, 2003, Oh et al., 1998, Werbonat et al., 2000), actin remodeling (Ochoa et al., 2000, Taylor et al., 2012) and migration (Razidlo et al., 2013). Dynamin selfassembles into tetramers which stimulates its basal GTPase activity (Warnock et al., 1996). The dynamin GTPase cycle is also regulated by post-translational modifications such as serine/threonine phosphorylation and subsequent dephosphorylation by calcineurin (Liu et al., 1994, Ahn et al., 1999, Graham et al., 2007). Dynamin is also phosphorylated at tyrosine residues by Src, which promotes its GTPase activity (Ahn et al., 2002). It was previously demonstrated that dynamin acts in a GTP-dependent manner to promote actin disassembly in osteoclasts (Ochoa et al., 2000). We also reported that shRNA-mediated knockdown of dynamin or over-expression of GTPase-inactive dynamin K44A in osteoclasts increased the thickness of the actin-rich podosome ring and decreased osteoclasts bone resorbing activity (Bruzzzniti, 2005). In the current study, we examined the expression of dynamin in osteoblasts, and demonstrated an important role for dynamin phosphorylation and GTPase activity in osteoblast differentiation and migration.

\section{Materials and Methods}

\subsection{Cell Culture}

Murine calvarial osteoblast cells were harvested from 2- to 3-day-old mice (Subramaniam et al., 2005) and digested in alpha-MEM containing $0.1 \%$ collagenase type $\mathrm{V}$ and $0.05 \%$ trypsin-EDTA for $15 \mathrm{~min}$ at $37^{\circ} \mathrm{C}$ with rotation. The cells were centrifuged and resuspended in alpha-MEM plus 10\% FBS and plated. Osteoclasts were prepared from murine bone marrow as previously described (Bruzzaniti et al., 2009). 293VnR cells (HEK293 human embryonic kidney cells stably expressing the vitronectin receptor) were described previously and validated as an appropriate model system to study dynamin GTPase activity (Bruzzaniti et al., 2005). MC3T3-E1 (ATCC) and 293VnR cells were transiently transfected in Opti-MEM media using FuGENE 6 as recommended by the manufacturer (Roche Diagnostics, Indianapolis, IN).

\subsection{Plasmids}

Dynamin 2, the most common dynamin isoform (Cook et al., 1994), was used for all of our studies. Rat dynamin 2 (splice variant aa) and dynK44A were inserted into the pEGFP-N1 vector as previously reported (Ochoa et al., 2000). The single point mutants (dynY231F, dynY597F) and double mutant (dynY231F/Y597F) were generated by site-directed mutagenesis (Bruzzaniti et al., 2005). Protein tyrosine phosphatase PTP-PEST containing a C-terminal myc tag was previously described (Davidson and Veillette, 2001). 


\subsection{Viral expression of dynamin and dynamin shRNA knockdown}

Recombinant adenovirus-expressing dynamin 2 (ba splice variant) (Damke et al., 2001) was used as previously reported (Bruzzaniti et al., 2005). Adenovirus expressing dynamin 2 shRNA or scrambled shRNA were generated using the Block-iT U6 RNAi entry vector kit and adenoviral RNAi expression system (Invitrogen) as reported (Bruzzaniti et al., 2009). The virus concentration used for dynamin over-expression or knockdown studies was optimized prior to use and a multiplicity of infection of 100-300 was used. After virus infection, calvarial osteoblasts were incubated in osteogenic media for up to 3 days prior to harvesting.

\subsection{Colorimetric dynamin GTP hydrolysis assay}

Dynamin GTPase activity was quantified using a modified non-radioactive in vitro GTP activity assay (Leonard et al., 2005). Briefly, dynamin was isolated from cells by immunoprecipitation (IP) with agarose beads. The IPs were washed 3 times with GTPase assay buffer (20 mM HEPES-KOH (pH 7.5), $20 \mathrm{mM} \mathrm{KCl,} 20 \mathrm{mM} \mathrm{MgCl}_{2}, 1 \mathrm{mM}$ DTT). Soluble GTP $(20 \mu \mathrm{M}$ final $)$ was then added to the agarose bead-protein complex and samples were incubated at $37^{\circ} \mathrm{C}$ for $1 \mathrm{hr}$. The supernatant $(5 \mu \mathrm{L})$ was transferred to a 96well microtiter plate containing $1.25 \mu \mathrm{L}$ of $0.5 \mathrm{M}$ EDTA. $100 \mu \mathrm{L}$ of Malachite green stock solution ( $1 \mathrm{mM}$ Malachite Green, and $10 \mathrm{mM}$ ammonium molybdate tetrahydrate) was added and color development was measured after 5-7 min at $650 \mathrm{~nm}$. The concentration of phosphate in solution was then calculated. A number of positive and negative controls were included; dynamin alone, GTP solution (substrate solution), empty protein G-agarose beads, RIPA buffer and un-transfected $293 \mathrm{VnR}$ cells were used. All background absorbance readings were subtracted from the absorbance values for the dynamin-containing samples. Our optimization studies demonstrated that the chemical components did not significantly contribute to the GTPase assay.

\subsection{Alkaline phosphatase activity}

Osteoblasts were cultured for up to 21 days in osteogenic media containing $10 \mu \mathrm{M}$ ascorbic acid and $50 \mu \mathrm{M} \beta$-glycerolphosphate. For alkaline phosphatase (ALP) staining, cells were fixed in $10 \%$ formalin for $15 \mathrm{~min}$. The ALP staining solution was prepared by dissolving 1 mg Naphthol AS=MX (Sigma) in one droplet of N,N-dimethylformamide (Wako, Osaka, Japan) and resuspended in $10 \mathrm{ml}$ of $0.1 \mathrm{M}$ Tris- $\mathrm{HCl}$ buffer containing $2 \mathrm{mM} \mathrm{MgCl} 2$. Fast BB salt (6 $\mu \mathrm{g}$, Sigma) was added. Cells were stained for $20 \mathrm{~min}$ at $37^{\circ} \mathrm{C}$, washed and stored dry. For ALP in vitro chemical assays, osteoblasts were suspended in $0.3 \mathrm{~mL}$ lysis buffer ( $0.1 \%$ triton $\mathrm{X}-100,50 \mathrm{mM} \mathrm{NaF}, 1 \%$ aprotinin, $1 \%$ pepstatin and $1 \%$ phenylmethanesulfonyl fluoride). An aliquot of cell lysate was added to ALP substrate buffer containing $2 \mathrm{mg} / \mathrm{mL}$ p-nitrophenyl phosphate in $1.5 \mathrm{M}$ alkaline buffer (Sigma), and the mixture was incubated at $37^{\circ} \mathrm{C}$ for $50 \mathrm{~min}$. The enzymatic reaction was stopped by the addition of $10 \mathrm{mM} \mathrm{NaOH}$, and the absorbance was read at $405 \mathrm{~nm}$. A protein assay was then performed using the BCA Protein Assay reagent (Pierce Biotechnology) and ALP activity was normalized to protein concentration.

\subsection{Migration assays}

Osteoblast migration assays were performed using Culture-Insert. $\mu$-Dishes as described by the manufacturer (Ibidi). Primary osteoblasts were seeded into the inner well of the $\mu$-Dish and incubated at $37^{\circ} \mathrm{C}$ and $5 \% \mathrm{CO}_{2}$. After overnight incubation, the insert was removed, unattached cells were rinsed off, and osteoblasts were incubated with alpha-MEM containing $0.5 \%$ serum in the presence of dynasore $(40 \mu \mathrm{M})$ or vehicle (DMSO) for $12 \mathrm{hrs}$. Alternatively, primary osteoblasts or MC3T3-E1 osteoblasts were transiently transfected and then plated onto coverslips. After $24 \mathrm{hrs,}$ a rubber policeman was used to remove cells from 
the center of the coverslip and the migration of cells into the clear zone was quantified microscopically. Images were taken using a Leica DMI4000B inverted microscope with attached digital camera. Osteoblasts were imaged using bright field or fluorescent microscopy (by virtue of a GFP tag) as necessary. Migration analyses were carried out using Image Pro software (Media Cybernetics, Inc. Bethesda, MD).

\subsection{Reverse transcription PCR and Quantitative PCR}

Complementary DNA (cDNA) was generated using the Transcriptor First Strand cDNA Synthesis Kit (Roche Applied Science, Mannheim, Germany). PCR was performed following standard protocols using oligonucleotide primers to dynamin (dynamin2) (5'TGGAGCCCGCATCAATCGTATCTT3' and 5'TTGCCTGACTCCGTGGATGTTCTT3'). GAPDH was used as an endogenous control (5'CTTTGGCATTGTGGAAGGGC3', 5'CAGGGATGATGTTCTGGGCA3'). PCR products were resolved on a $2 \%$ agarose gel containing ethidium bromide and imaged. Alternatively, Syber® green Gene Expression Master Mix (Applied Biosystems, Warrington, UK) was used for quantitative PCR (QPCR). Samples were analyzed in the Applied Biosystems QPCR machine using Step-One Software. The threshold cycle (Ct) for each test gene was normalized against their respective endogenous controls. QPCR was analyzed for fold change in expression relative to controls with $\Delta \mathrm{Ct}$ values of the sample and reference gene using the formula 2- $\Delta \Delta \mathrm{Ct}$. Samples were prepared in duplicate and experiments were repeated 3 times and the mean and SEM calculated. The QPCR primers used to detect osteoblast genes were as follows; c-fos ( $5^{\prime}$ ACTTCTTGTTTCCGGC3', 5'AGCTTCAGGGTAGGTG3'), type I collagen (5'AACCTGGTGCGAAAGGTGAA3', 5'AGGAGCACCAACGTTACCAA3'), osteocalcin (5'TCTCTCTGACCTCACAGATGCCAAGC3', 5'GGACTGAGGCTCCAAGGTAGCG3') and osterix (5'CCCTTCTCAAGCACCAATGG3', 5'AGGGTGGGTAGTCATTTGCATAG3'). The housekeeping gene 18S RNA was used the internal reference control and amplified using the 5'AGTCCCTGCCCTTTGTACACA $3^{\prime}$ and $5^{\prime}$ CGATCCGAGGGCCTCACTA3' primers.

\section{Results}

\subsection{Dynamin is expressed during osteoblast differentiation}

We first examined if dynamin was expressed in primary osteoblasts, and whether the expression of dynamin was altered during osteoblast differentiation. Primary osteoblasts were isolated from the calvaria of neonatal mice and total cell lysates (TCL) were subject to SDS-PAGE and Western blotting with an antibody to endogenous dynamin. Since we previously demonstrated osteoclasts express dynamin (Bruzzaniti et al., 2005), we used primary murine osteoclasts as a positive control (Fig. 1A). Western blotting confirmed that dynamin is endogenously expressed in osteoblasts, as well as osteoclasts. We next differentiated osteoblasts for 0-21 days in the presence of osteogenic media containing ascorbic acid and $\beta$-glycerolphosphate, and then examined dynamin mRNA levels on different days using quantitative real-time PCR (QPCR) (Fig. 1B). Dynamin mRNA was detected throughout the osteoblast differentiation process, with an increase in dynamin levels detected in day 7 cultures compared to day 3 cultures. These findings suggested that dynamin may play a role in osteoblast differentiation and function.

\subsection{Osteoblast differentiation is regulated by dynamin activity}

We examined if dynamin played a functional role in osteoblasts by examining the effect of dynamin on alkaline phosphatase (ALP) activity. ALP is a key enzyme expressed early in the osteoblasts differentiation process that regulates calcium phosphate deposition (Anh et al., 1998, Bonucci and Nanci, 2001). We over-expressed dynamin in calvarial-derived 
osteoblasts using adenovirus-mediated infection. Infected osteoblasts expressing dynamin or a control were differentiated in osteogenic media containing ascorbic acid and $\beta$ -

glyerolphosphate for 3 days then either stained for ALP or assayed for ALP activity using an in vitro biochemical assay. As shown in Fig. $2 \mathrm{~A}$, overexpression of dynamin, but not the control virus, significantly reduced ALP staining and ALP activity in osteoblasts.

Alternatively, we examined the effect of dynamin knockdown on ALP expression and activity. Calvarial osteoblasts were infected with adenoviruses expressing dynamin short hairpin RNA (shRNA) or a scrambled shRNA. Cells were also differentiated in osteogenic media for 3 days prior to ALP assay. Dynamin shRNA knockdown reduced dynamin levels by approximately $50 \%$ and led to an increase in ALP staining and ALP activity in osteoblasts (approximately 2-fold increase) (Fig. 2B), further supporting a role for dynamin in osteoblast differentiation.

We next examined if dynamin GTPase activity was important for its effect on ALP activity. We used MC3T3-E1 osteoblast cells which can be differentiated into mature osteoblasts in the presence of osteogenic media. MC3T3-E1 cells were incubated for 1, 2 or 3 days in osteogenic media containing dynasore, a specific chemical inhibitor of dynamin GTPase activity (Kirchhausen et al., 2008, Macia et al., 2006) (Fig. 2C). Osteoblasts treated with/ without dynasore were collected each day and assayed for ALP activity. Of interest, dynasore stimulated ALP activity in a dose- and time-dependent manner, with maximal ALP activity observed after 1 day using low concentrations of dynasore (5 and $15 \mu \mathrm{M})$. In contrast, higher concentrations of dynasore $(40 \mu \mathrm{M})$ inhibited ALP activity after 24 hrs. After 3 days, the magnitude of the dynasore response was reduced for all concentrations tested, although a higher level of ALP activity was still observed in cells treated with $15 \mu \mathrm{M}$ dynasore. To confirm these results were not due to decreases in cell viability, we used the trypan-blue exclusion viability test. Cell viability for all dynasore-treated osteoblasts was found to be greater than $97 \%$ (data not shown). These studies suggest that inhibition of dynamin GTPase activity promotes ALP activity in a dose-dependent and time-dependent manner, having its greatest stimulatory effect in early osteoblast cultures.

To further examine the effect of dynamin inhibition on osteoblast differentiation, we treated primary osteoblasts for 24 hours with dynasore and then performed QPCR analysis of known osteoblast transcription factors or functional proteins as follows; proliferating/preosteoblasts (c-fos), pre-osteoblasts to osteoblast transition (osterix) and osteoblasts/mature osteoblasts (type I collagen, osteocalcin) (Raisz, 1999) (Fig. 2D). These studies revealed a significant decrease in c-fos and osterix expression in osteoblasts treated with dynasore, compared to vehicle-treated cells. However, collagen and osteocalcin mRNA levels remained unchanged after dynasore treatment. Since c-fos and osterix expression precede ALP expression during osteoblast differentiation, these studies suggest that inhibition of dynamin GTPase activity promotes the differentiation of early osteoblasts towards increased ALP activity. However, dynasore does not appear to affect the differentiation of osteoblasts into mature cells expressing both type I collagen or osteocalcin.

\subsection{PTP-PEST decreases the phosphorylation and GTPase activity of dynamin}

Tyrosine phosphorylation is known to regulate dynamin GTPase activity (Ahn et al., 2002), but the phosphatase involved in dephosphorylating dynamin is unknown. PTP-PEST is a ubiquitously-expressed cytosolic phosphatase that is involved in tyrosine dephosphorylation (Alonso et al., 2004, Veillette et al., 2009, Eleniste et al., 2012, Chellaiah et al., 2007). We first examined if dynamin could form a complex with PTP-PEST in osteoblasts and if dynamin GTPase activity was necessary for their interaction. Primary murine osteoblasts were treated with dynasore or a vehicle control and subject to immunoprecipitation (IPs) with antibodies to either dynamin or PTP-PEST. Using reciprocal antibody combinations for 
IPs we found that endogenous dynamin and PTP-PEST formed a protein complex in osteoblasts (Fig. 3A). Moreover, dynasore had little effect on the association of dynamin with PTP-PEST in osteoblasts. To confirm if endogenous dynamin was dephosphorylated by phosphatases, calvarial osteoblasts were subject to IP using an antibody to dynamin, followed by Western blotting using an antibody that detects phosphorylated tyrosine residues (p-tyr). IPs were also performed with an IgG antibody to confirm the absence of non-specific binding. To stabilize phosphorylated dynamin levels, osteoblasts were treated with the phosphatase inhibitor phenylarsine oxide (PAO), which is a known inhibitor of PTP-PEST (Chellaiah et al., 2007) (Fig. 3B). Although we detected a low level of phosphorylated dynamin in osteoblasts, we found an increase in phosphorylated dynamin with increasing concentrations of PAO, suggesting that the tyrosine dephosphorylation of dynamin in osteoblasts may be tightly regulated by phosphatases.

We next determined if PTP-PEST was involved in dynamin dephosphorylation, and if dephosphorylation affected dynamin GTPase activity. Because of the low transfection efficiency of osteoblast, dynWT and PTP-PEST were co-expressed in 293VnR cells which we previously validated as an appropriate model system for dynamin activity assay. Changes in dynamin phosphorylation were then examined by IP and Western blotting with a phospho-tyrosine specific antibody, followed by densitometric analysis of blots. Similar to our findings in osteoblasts, dynamin co-immunoprecipitated with PTP-PEST in 293VnR cells (Fig. 3C). In the presence of PTP-PEST, we also found that the level of phosphorylated dynamin significantly decreased by $75-90 \%$, compared cells expressing dynWT only. Like PAO, sodium orthovanadate $\left(\mathrm{Na}_{3} \mathrm{VO}_{4}\right)$ is a known chemical inhibitor of PTP-PEST phosphatase activity. We examined if inhibition of transfected PTP-PEST by $\mathrm{Na}_{3} \mathrm{VO}_{4}$ could restore dynamin phosphorylation and consequently dynamin GTPase activity. We treated cells expressing dynWT and PTP-PEST with the $\mathrm{Na}_{3} \mathrm{VO}_{4}$ for 2 and 3 hours and examined dynamin phosphorylation (Fig. 3D) and GTPase activity (see below). We found a $62-85 \%$ increase in dynamin phosphorylation in cells treated with $\mathrm{Na}_{3} \mathrm{VO}_{4}$, compared to vehicletreated cells. Similar results were found using PAO (data not shown). Indeed, $\mathrm{Na}_{3} \mathrm{VO}_{4}$ completely reversed the effects of PTP-PEST on dynamin phosphorylation levels after 2 hours.

We next examined if dynamin dephosphorylation by PTP-PEST negatively regulated dynamin GTPase activity. In order to quantitate dynamin GTPase activity we used a modified in vitro colorimetric dynamin GTPase assay. The dynamin GTPase activity assay was based on the ability of immunoprecipitated dynamin to hydrolyze GTP to GDP, releasing inorganic phosphate $\left(\mathrm{P}_{\mathrm{i}}\right)$ (Leonard et al., 2005). The dynamin GTPase activity assay was validated using a number of positive and negative controls. Positive controls (dynWT) showed high levels of GTPase activity. Negative controls, including dynasore and dynK44A, a known GTPase-inactive mutant) (Damke et al., 1994, Song et al., 2004, Kirchhausen et al., 2008) showed low or undetectable levels of GTPase activity as expected (data not shown). Using this assay, we examined the effect of PTP-PEST activity on dynamin GTPase activity. Aliquots of the same cell lysates prepared for IPs (see Fig. 3C) were used for these analyses and Western blotting confirmed similar expression levels of dynamin in each sample. We observed a corresponding 78-88\% decrease in dynWT GTPase activity with increasing expression of PTP-PEST (Fig. 3E). Since the phosphorylation of dynamin was increased in a time-dependent manner by the phosphatase inhibitor $\mathrm{Na}_{3} \mathrm{VO}_{4}$, we examined its ability to restore dynamin GTPase activity in the presence of PTP-PEST. Consistent with the effect of $\mathrm{Na}_{3} \mathrm{VO}_{4}$, on dynamin phosphorylation, a time-dependent increase in dynamin GTPase activity was also observed in the presence of $\mathrm{Na}_{3} \mathrm{VO}_{4}$, (Fig. 3F). Indeed, $\mathrm{Na}_{3} \mathrm{VO}_{4}$ increased dynamin GTPase activity above the levels of the dynamin only control, most likely as a result of inhibition of endogenous as well as over-expressed phosphatase activity. Together, these results suggest that PTP-PEST promotes dynamin 
dephosphorylation at tyrosine residues, leading to a corresponding decrease in dynamin GTPase activity.

\subsection{PTP-PEST reverses the effect of Src on dynamin phosphorylation and activity}

It was previously demonstrated that dynamin phosphorylation at tyrosine residues occurs in part by c-Src (Foster-Barber and Bishop, 1998, Ahn et al., 1999, Ahn et al., 2002). To determine if PTP-PEST-mediated dephosphorylation of dynamin occurred at Src target sites, dynamin and c-Src were expressed in the presence or absence of PTP-PEST. Dynamin was subject to IP and analyzed for phosphorylation by Western blotting and densitometry (Fig. $4 \mathrm{~A})$. We found that c-Src catalyzed the tyrosine phosphorylation of dynamin, increasing its phosphorylation level by $130 \%$, compared to controls. However, in cells containing dynWT, Src and PTP-PEST, the level of phosphorylated dynamin was decreased to $30 \%$ of controls $(\mathrm{dyn}+\mathrm{Src})$. We also examined the effect of PTP-PEST on Src-mediated increases in dynamin GTPase activity (Fig. 4B). In parallel to changes in dynamin phosphorylation, we observed a corresponding increase in dynamin GTPase activity in the presence of Src, which was reversed by the addition of PTP-PEST.

Two putative Src phosphorylation sites have been reported in dynamin; Y231 and Y597 (Foster-Barber and Bishop, 1998, Ahn et al., 1999, Ahn et al., 2002). We confirmed the importance of these tyrosine sites for overall dynamin phosphorylation and GTPase activity by expressing Src and PTP-PEST with dynY231F, dynY597F or dynY231F/Y597F. Dynamin was IP and analyzed for phosphorylation by Western blotting and densitometry. As expected, the phosphorylation level of dynY231F, dynY597F and dynY231F/Y597F were significantly lower $(75 \%, 65 \%$ and $70 \%$, respectively) than dynWT, and were similar to cells expressing dynWT + PTP-PEST. We also observed a robust $70 \%$ decrease in the GTPase activity of dynY231F, dynY597F and dynY231F/Y597F, compared to dynWT (Fig. 4D). Co-expression of PTP-PEST with dynY231F, dynY597F or dynY231F/Y597F had only a minor inhibitory effect on the dephosphorylation or GTPase-activity of the dynamin tyrosine mutants as anticipated. These findings confirmed that Y231 and Y597 significantly contribute to the overall tyrosine phosphorylation state of dynamin, which regulates dynamin GTPase activity.

\subsection{Effect of dynamin phosphorylation and GTPase activity on osteoblast function}

To examine if dynamin phosphorylation was important for ALP activity, we expressed dynWT, dynK44A (GTPase-defective) or dynY231F/Y597F (phosphorylation-defective) in MC3T3-E1 osteoblasts and then assayed ALP activity. Western blotting confirmed a similar level of expression of dynWT, dynK44A and dynY231F/Y597F in osteoblasts (Fig. 5). DynK44A increased ALP expression in MC3T3-E1 cells by almost 80\%, compared to dynWT. Although dynY231F/Y597F-expressing osteoblasts exhibited a small increase in ALP activity (40\% increase compared to dynWT), the overall effect of dynY231F/Y597F on ALP activity was significantly less than that of dynK44A.

Since osteoblasts are migratory cells that are recruited to resorbed bone surfaces, we examined whether dynamin activity and phosphorylation played a role in osteoblast migration. Calvarial osteoblasts were plated in migration chambers and treated with or without dynasore $(5$ or $40 \mu \mathrm{M})$ and imaged at 0 and $12 \mathrm{hrs} \mathrm{(Fig.} \mathrm{6A).} \mathrm{Osteoblasts} \mathrm{treated}$ with $5 \mu \mathrm{M}$ dynasore displayed a similar migration pattern as vehicle treated cells. However, higher doses of dynasore $(40 \mu \mathrm{M})$ led to a significant decrease $(-50 \%)$ in osteoblast migration, compared to vehicle-treated controls. Dynasore is reported to be a specific chemical inhibitor of dynamin (Kirchhausen et al., 2008, Macia et al., 2006). However, we cannot yet exclude the possibility that the decrease in migration in the presence of $40 \mu \mathrm{M}$ dynasore was due in part to inhibition of other GTPases in osteoblasts. To further examine 
the role of dynamin phosphorylation in the osteoblast migration process, we expressed dynWT, dynK44A or dynY231F/Y597F in MC3T3-E1 osteoblastic cells. All transfected proteins contained a GFP-tag and therefore could be imaged under fluorescence microscopy (Fig. 6B). The expression of dynWT had no significant effect on osteoblast migration, perhaps reflecting the fact that MC3T3-E1 cells express endogenous dynamin and that these cells are already highly migratory. Nevertheless, as expected over-expression of dynK44A inhibited MC3T3-E1 migration by approximately 40\%. Furthermore, the dynY231F/Y597F mutant inhibited osteoblast migration to a much greater extent than dynK44A, resulting in a $62 \%$ decrease in migration. Together, these findings were consistent with the inhibitory effect of dynasore on migration, and suggested that osteoblast migration is regulated by both the tyrosine phosphorylation and GTPase activity of dynamin.

\section{Discussion}

Osteoblasts are derived from the mesenchymal stem cell pool and differentiate from preosteoblasts into mature bone-forming osteoblast through the tight regulatory control of transcription factors and functional proteins. We previously demonstrated that dynamin GTPase activity is critical for promoting the bone-resorbing function of osteoclasts, and that inhibition of dynamin blocked osteoclast function. Our current studies now demonstrate an important role for dynamin in osteoblast differentiation and migration. Dynamin expression was found to be temporally regulated during osteoblast differentiation, with the highest level dynamin mRNA detected in 0-7 day cultures. We also found that the expression of dynamin in osteoblasts negatively regulated ALP activity, a marker of early osteoblast differentiation. Specifically, over-expression of dynamin reduced ALP levels, whereas shRNA-mediated knockdown of dynamin stimulated ALP activity. The important role of dynamin GTPase activity on ALP expression was also confirmed using the dynamin inhibitor dynasore, albeit at low concentrations, and by over-expression of kinase-defective dynK44A in MC3T3-E1 osteoblast cells. In contrast to dynK44A, dynY231F/Y597F modestly increased ALP activity in MC3T3-E1 cells, despite similar levels of expression, which may reflect the different rates of GTP-hydrolysis by these mutants. The increase in ALP activity in osteoblasts treated with 5 and $15 \mu \mathrm{M}$ dynasore also correlated with decreased c-fos and osterix mRNA levels, which are involved in osteoblast proliferation and pre-osteoblastosteoblast transition, respectively. Together, these findings suggest that inhibition of dynamin promotes the differentiation of osteoblasts from pre-osteoblasts into early differentiated osteoblasts, as evidenced by increased ALP activity. However, it appears that dynamin activity is dispensable for latter stages of osteoblast differentiation as indicated by the finding that long-term incubation with dynasore ( 3 days) had little effect on ALP expression, and collagen and osteocalcin mRNA levels were unaffected by dynasore. In studies published by others (Heining et al., 2011), it was reported that $40 \mu \mathrm{M}$ dynasore blocked the ability of the bone morphogenic protein 2 (BMP-2) to induce transcription in the osteoblastic cell line, $\mathrm{C} 2 \mathrm{C} 12$, which was suggested to attenuate osteoblast differentiation. However, no dose-response analyses were performed, and we found a clear temporal and dose-dependent effect of dynasore on osteoblast differentiation.

Dynamin GTPase activity is regulated by self-assembly, intracellular localization and posttranslational modification (Warnock et al., 1996, Schafer, 2004, Shajahan et al., 2004). Dynamin contains several potential tyrosine phosphorylation sites (Cook et al., 1994), but the cellular effect of dynamin phosphorylation is poorly understood. Our study revealed low levels of phosphorylated dynamin in osteoblasts under basal conditions (Fig. 3). However, inhibition of phosphatase activity increased the level of phosphorylated dynamin, suggesting tyrosine phosphorylation/dephosphorylation in osteoblasts is a transient and tightlyregulated event. We also found that endogenous dynamin forms a protein complex with the tyrosine phosphatase PTP-PEST in osteoblasts. In addition, transient expression of PTP- 
PEST with dynamin led to a dose-dependent decrease in dynamin phosphorylation, which was highly correlated with a decrease in dynamin GTPase activity. PTP-PEST was also found to reverse the Src-mediated phosphorylation of dynamin, which promoted dynamin GTPase activity (Fig. 4). Whether the association of dynamin with PTP-PEST is direct or mediated by other proteins is not yet known. Moreover, our findings do not exclude the possibility that other phosphatases present in osteoblasts may participate in the dephosphorylation of dynamin. Nevertheless, our studies suggest that the dephosphorylation of dynamin may in part be regulated by PTP-PEST, and that dynamin dephosphorylation is important for restoring dynamin GTPase activity to its basal state.

Previously, it was shown that tyrosine residues Y231 and Y597 are phosphorylated in response to $\beta_{2}$-adrenergic stimulation (Ahn et al., 1999) and are necessary for epidermal growth factor receptor internalization (Ahn et al., 2002). It has also been shown that c-Src is involved in the tyrosine phosphorylation of dynamin at residue Y597 (Foster-Barber and Bishop, 1998, Ahn et al., 1999, Ahn et al., 2002). Consistent with a decrease in the phosphorylation level of dynY231F, dynY597F and dynY231F/Y597F, the GTPase activity of these tyrosine mutants was also significantly lower than dynWT (Fig. 4), confirming that dynamin phosphorylation is a critical step for GTPase activation. Moreover, mutation of these sites affected the ability of PTP-PEST to dephosphorylate dynamin. Given that dynamin self-assembly and phosphorylation stimulate its basal GTPase activity, we speculate that the dephosphorylation of dynamin is likely to occur after self-assembly stimulated GTP-hydrolysis, or alternatively, that dephosphorylation by PTP-PEST or other phosphatases reduces the ability of dynamin to self-assemble.

During bone remodeling, osteoblasts are recruited to eroded bone surfaces where they begin the process of bone formation. Our finding that dynamin regulates ALP activity, a marker of early osteoblasts, led us to also examine the role of dynamin in osteoblast migration. Our studies demonstrated that dynasore $(40 \mu \mathrm{M})$ and dynK44A significantly inhibited osteoblast cell migration (Fig. 6). Of interest, the phosphorylation mutant dynY231F/Y597F blocked osteoblast migration to a greater extent than dynK44A, but had a lesser effect on ALP activity. These findings suggested that tyrosine phosphorylation of dynamin is an important step in the migration of osteoblasts, which is likely to affect dynamin's conformation and its binding to downstream signaling proteins (Wang et al., 2011, Krause et al., 2003, Nagano et al., 2012). Consistent with this hypothesis, it was recently shown in tumor cells that dynamin Y231 is phosphorylated at focal adhesions by Src, and that dynamin is recruited to focal adhesions by the focal adhesion kinase FAK (Wang et al., 2011). A link between dynamin and actin turnover has also been shown in osteoclasts (Ochoa et al., 2000; Bruzzaniti et al., 2005) and osteoblasts (Eleniste \& Bruzzaniti et al., 2012 and unpublished data). However, additional studies are needed to better understand the role of dynamin in regulating focal adhesion turnover rate and its effect on cell migration.

In summary, our results demonstrate that dynamin GTPase activity, which is regulated in part by changes in its phosphorylation, controls the differentiation and migration of osteoblasts. Our findings also demonstrate that inhibition of dynamin GTPase activity promoted early osteoblast differentiation leading to an increase in ALP activity, and led to a decrease in osteoblast migration. Interestingly, we also previously reported that inhibition of dynamin blocked both osteoclast bone resorbing activity and osteoclast migration on dentin slices (Bruzzaniti et al., 2005) (see Figure 6C). Taken together, our results suggest that inhibitors of dynamin may be useful for the treatment of bone loss diseases by promoting early osteoblast activity while inhibiting osteoclast bone resorbing activity. 


\section{Acknowledgments}

We thank Dr. A. Veillette (Montréal, Québec), for providing PTP-PEST and Dr. Sandra Schmid (La Jolla, CA, USA) for dynamin adenoviruses.

\section{Funding}

This work was funded in part by a NIH/NIAMS R01 grant (\#AR060332), a CTSI Collaboration in Biomedical/ Translational Research Grant (NIH \#RR025761) and by funds from the Indiana University School of Dentistry to A.B.

\section{Abbreviations}

$\begin{array}{ll}\text { IP } & \text { immunoprecipitation } \\ \text { co-IP } & \text { co-immunoprecipitation } \\ \text { B } & \text { Western blot } \\ \text { dyn } & \text { dynamin } \\ \text { TCL } & \text { total cell lysate } \\ \text { GFP } & \text { green fluorescent protein }\end{array}$

\section{References}

Ahn S, Kim J, Lucaveche CL, Reedy MC, Luttrell LM, Lefkowitz RJ, Daaka Y. Src-dependent tyrosine phosphorylation regulates dynamin self-assembly and ligand-induced endocytosis of the epidermal growth factor receptor. J Biol Chem. 2002; 277:26642-26651. [PubMed: 12011079]

Ahn S, Maudsley S, Luttrell LM, Lefkowitz RJ, Daaka Y. Src-mediated tyrosine phosphorylation of dynamin is required for beta2-adrenergic receptor internalization and mitogen-activated protein kinase signaling. J Biol Chem. 1999; 274:1185-1188. [PubMed: 9880482]

Alonso A, Sasin J, Bottini N, Friedberg I, Friedberg I, Osterman A, Godzik A, Hunter T, Dixon J, Mustelin T. Protein tyrosine phosphatases in the human genome. Cell. 2004; 117:699-711. [PubMed: 15186772]

Anh DJ, Dimai HP, Hall SL, Farley JR. Skeletal alkaline phosphatase activity is primarily released from human osteoblasts in an insoluble form, and the net release is inhibited by calcium and skeletal growth factors. Calcif Tissue Int. 1998; 62:332-340. [PubMed: 9504959]

Bonucci E, Nanci A. Alkaline phosphatase and tartrate-resistant acid phosphatase in osteoblasts of normal and pathologic bone. Ital J Anat Embryol. 2001; 106:129-133. [PubMed: 11729947]

Bruzzaniti A, Neff L, Sandoval A, Du L, Horne WC, Baron R. Dynamin reduces Pyk2 Y402 phosphorylation and SRC binding in osteoclasts. Mol Cell Biol. 2009; 29:3644-3656. [PubMed: 19380485]

Bruzzaniti A, Neff L, Sanjay A, Horne WC, De Camilli P, Baron R. Dynamin forms a Src kinasesensitive complex with $\mathrm{Cbl}$ and regulates podosomes and osteoclast activity. Mol Biol Cell. 2005; 16:3301-3313. [PubMed: 15872089]

Chellaiah MA, Kuppuswamy D, Lasky L, Linder S. Phosphorylation of a Wiscott-Aldrich syndrome protein-associated signal complex is critical in osteoclast bone resorption. J Biol Chem. 2007; 282:10104-10116. [PubMed: 17283076]

Cook TA, Urrutia R, McNiven MA. Identification of dynamin 2, an isoform ubiquitously expressed in rat tissues. Proc Natl Acad Sci U S A. 1994; 91:644-648. [PubMed: 8290576]

Damke H, Baba T, Warnock DE, Schmid SL. Induction of mutant dynamin specifically blocks endocytic coated vesicle formation. J Cell Biol. 1994; 127:915-934. [PubMed: 7962076]

Damke H, Binns DD, Ueda H, Schmid SL, Baba T. Dynamin GTPase domain mutants block endocytic vesicle formation at morphologically distinct stages. Mol Biol Cell. 2001; 12:2578-2589. [PubMed: 11553700] 
Davidson D, Veillette A. PTP-PEST, a scaffold protein tyrosine phosphatase, negatively regulates lymphocyte activation by targeting a unique set of substrates. EMBO J. 2001; 20:3414-3426. [PubMed: 11432829]

Ducy P, Schinke T, Karsenty G. The osteoblast: a sophisticated fibroblast under central surveillance. Science. 2000; 289:1501-1504. [PubMed: 10968779]

Eleniste PP, Du L, Shivanna M, Bruzzaniti A. Dynamin and PTP-PEST cooperatively regulate Pyk2 dephosphorylation in osteoclasts. Int J Biochem Cell Biol. 2012; 44:790-800. [PubMed: 22342188]

Eleniste PP, Bruzzaniti A. Focal adhesion kinases in adhesion structures and disease. J Signal Transduct. 2012; 2012:296450. [PubMed: 22888421]

Foster-Barber A, Bishop JM. Src interacts with dynamin and synapsin in neuronal cells. Proc Natl Acad Sci U S A. 1998; 95:4673-4677. [PubMed: 9539797]

Graham ME, Anggono V, Bache N, Larsen MR, Craft GE, Robinson PJ. The in vivo phosphorylation sites of rat brain dynamin I. J Biol Chem. 2007; 282:14695-14707. [PubMed: 17376771]

Heining E, Bhushan R, Paarmann P, Henis YI, Knaus P. Spatial segregation of BMP/Smad signaling affects osteoblast differentiation in C2C12 cells. PLoS One. 2011; 6(10):e25163. [PubMed: 21998639]

Hinshaw JE. Dynamin and its role in membrane fission. Annu Rev Cell Dev Biol. 2000; 16:483-519. [PubMed: 11031245]

Kirchhausen T, Macia E, Pelish HE. Use of dynasore, the small molecule inhibitor of dynamin, in the regulation of endocytosis. Methods Enzymol. 2008; 438:77-93. [PubMed: 18413242]

Krause M, Dent EW, Bear JE, Loureiro JJ, Gertler FB. Ena/VASP proteins: regulators of the actin cytoskeleton and cell migration. Annu Rev Cell Dev Biol. 2003; 19:541-564. [PubMed: 14570581]

Leonard M, Song BD, Ramachandran R, Schmid SL. Robust colorimetric assays for dynamin's basal and stimulated GTPase activities. Methods Enzymol. 2005; 404:490-503. [PubMed: 16413294]

Liu JP, Sim AT, Robinson PJ. Calcineurin inhibition of dynamin I GTPase activity coupled to nerve terminal depolarization. Science. 1994; 265:970-973. [PubMed: 8052858]

Macia E, Ehrlich M, Massol R, Boucrot E, Brunner C, Kirchhausen T. Dynasore, a cell-permeable inhibitor of dynamin. Dev Cell. 2006; 10:839-850. [PubMed: 16740485]

Mackie EJ. Osteoblasts: novel roles in orchestration of skeletal architecture. Int J Biochem Cell Biol. 2003; 35:1301-1305. [PubMed: 12798343]

Nagano M, Hoshino D, Koshikawa N, Akizawa T, Seiki M. Turnover of focal adhesions and cancer cell migration. Int J Cell Biol. 2012; 2012:310616. [PubMed: 22319531]

Ochoa GC, Slepnev VI, Neff L, Ringstad N, Takei K, Daniell L, Kim W, Cao H, McNiven M, Baron $\mathrm{R}$, De Camilli P. A functional link between dynamin and the actin cytoskeleton at podosomes. J Cell Biol. 2000; 150:377-389. [PubMed: 10908579]

Oh P, McIntosh DP, Schnitzer JE. Dynamin at the neck of caveolae mediates their budding to form transport vesicles by GTP-driven fission from the plasma membrane of endothelium. J Cell Biol. 1998; 141:101-114. [PubMed: 9531551]

Raisz LG. Physiology and pathophysiology of bone remodeling. Clin Chem. 1999; 45:1353-1358. [PubMed: 10430818]

Razidlo GL, Wang Y, Chen J, Krueger EW, Billadeau DD, McNiven MA. Dynamin 2 potentiates invasive migration of pancreatic tumor cells through stabilization of the Rac1 GEF Vav1. Dev Cell. 2013; 24:573-585. [PubMed: 23537630]

Schafer DA. Regulating actin dynamics at membranes: a focus on dynamin. Traffic. 2004; 5:463-469. [PubMed: 15180823]

Schmid SL, McNiven MA, De Camilli P. Dynamin and its partners: a progress report. Curr Opin Cell Biol. 1998; 10:504-512. [PubMed: 9719872]

Shajahan AN, Timblin BK, Sandoval R, Tiruppathi C, Malik AB, Minshall RD. Role of Src-induced dynamin-2 phosphorylation in caveolae-mediated endocytosis in endothelial cells. J Biol Chem. 2004; 279:20392-20400. [PubMed: 15007081] 
Song BD, Leonard M, Schmid SL. Dynamin GTPase domain mutants that differentially affect GTP binding, GTP hydrolysis, and clathrin-mediated endocytosis. J Biol Chem. 2004; 279:4043140436. [PubMed: 15262989]

Song BD, Schmid SL. A molecular motor or a regulator? Dynamin's in a class of its own. Biochemistry. 2003; 42:1369-1376. [PubMed: 12578348]

Subramaniam M, Gorny G, Johnsen SA, Monroe DG, Evans GL, Fraser DG, Rickard DJ, Rasmussen K, van Deursen JM, Turner RT, Oursler MJ, Spelsberg TC. TIEG1 null mouse-derived osteoblasts are defective in mineralization and in support of osteoclast differentiation in vitro. Mol Cell Biol. 2005; 25:1191-1199. [PubMed: 15657444]

Tanaka Y, Nakayamada S, Okada Y. Osteoblasts and osteoclasts in bone remodeling and inflammation. Curr Drug Targets Inflamm Allergy. 2005; 4:325-328. [PubMed: 16101541]

Taylor MJ, Lampe M, Merrifield CJ. A feedback loop between dynamin and actin recruitment during clathrin-mediated endocytosis. PLoS Biol. 2012; 10:e1001302. [PubMed: 22505844]

Veillette A, Rhee I, Souza CM, Davidson D. PEST family phosphatases in immunity, autoimmunity, and autoinflammatory disorders. Immunol Rev. 2009; 228:312-324. [PubMed: 19290936]

Wang Y, Cao H, Chen J, McNiven MA. A direct interaction between the large GTPase dynamin-2 and FAK regulates focal adhesion dynamics in response to active Src. Mol Biol Cell. 2011; 22:15291538. [PubMed: 21411625]

Warnock DE, Hinshaw JE, Schmid SL. Dynamin self-assembly stimulates its GTPase activity. J Biol Chem. 1996; 271:22310-22314. [PubMed: 8798389]

Werbonat Y, Kleutges N, Jakobs KH, van Koppen CJ. Essential role of dynamin in internalization of M2 muscarinic acetylcholine and angiotensin AT1A receptors. J Biol Chem. 2000; 275:2196921974. [PubMed: 10801820] 
A.

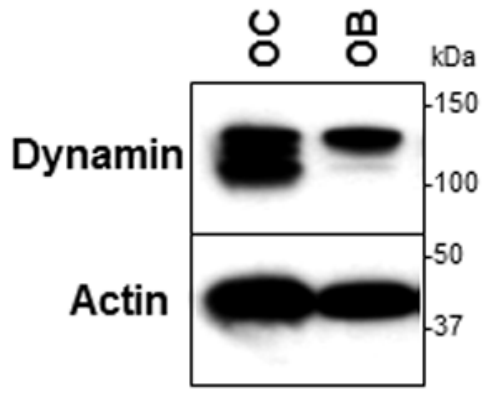

B.

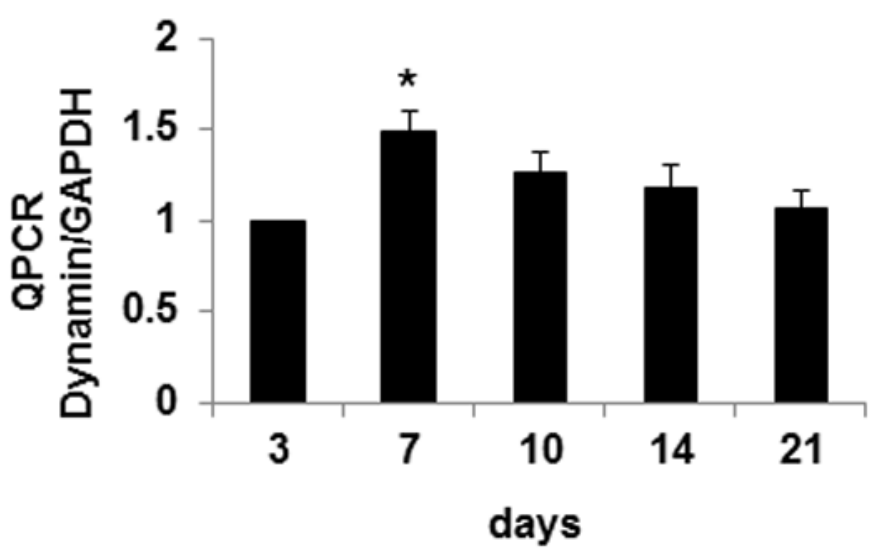

Fig. 1. Dynamin is expressed during osteoblast differentiation

(A) Total cell lysates (TCL) from murine-derived osteoclasts and calvarial osteoblasts were blotted for dynamin. Equal protein loading was confirmed with actin. Protein molecular (kDa) markers are shown. (B) Calvarial osteoblasts were differentiated in osteogenic media for 0-21 days. Quantitative real-time PCR was performed using primers specific to dynamin and results were expressed relative to dynamin levels at day 3. GAPDH was used as the reference mRNA control. Samples were prepared in triplicate and average \pm SEM is shown. Experiments were reproduced 3 times. Statistical significance was performed using Student's t-Test and significance $(\mathrm{p}<0.05)$ relative to day 3 is indicated by the asterisk $(*)$. 
A.

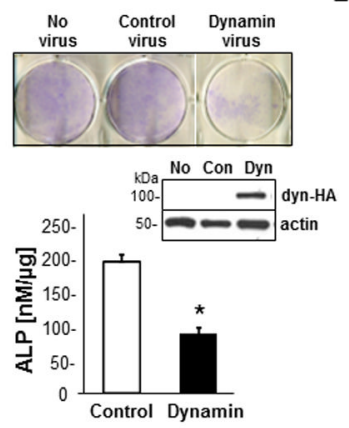

B.

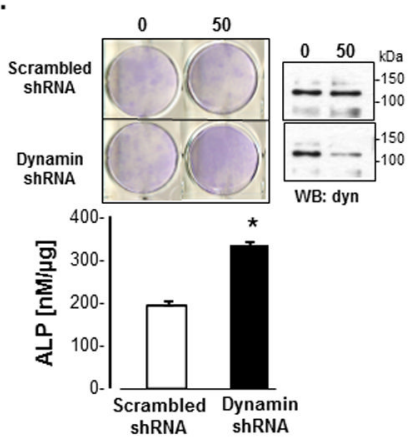

c.

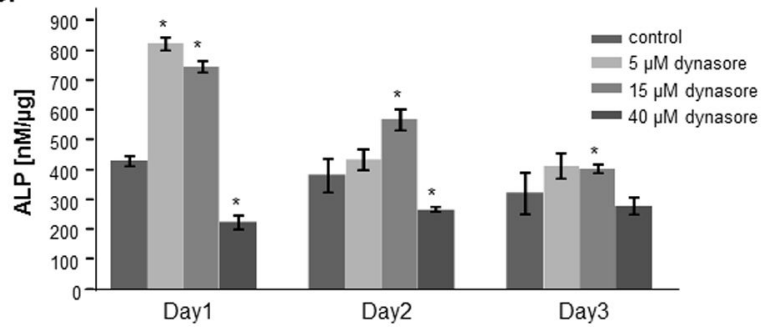

D.

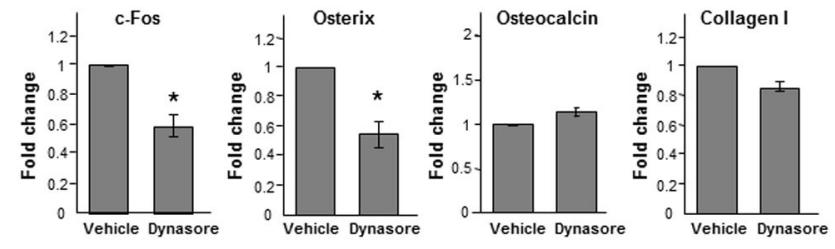

Fig. 2. Dynamin regulates alkaline phosphatase activity

(A) Calvarial osteoblasts were infected with adenoviruses expressing dynamin (HA-tagged) or control (con) virus and incubated in osteogenic media for 3 days, then fixed and stained for ALP activity. Pre-optimized virus concentrations were used. Representative ALP stained osteoblasts and Western blot (WB) demonstrating increased dynamin-HA levels are shown. Protein loading was normalized by blotting for actin. ALP activity was also quantified using an in vitro assay and results were normalized for total protein. (B) Osteoblasts were infected with dynamin shRNA adenovirus or a control scrambled shRNA virus, differentiated for 3 days in osteogenic media and stained or assayed for ALP activity. Dynamin knockdown was confirmed by Western blotting with an antibody to total dynamin. (C) Calvaria-derived osteoblasts were differentiated with increasing concentrations of dynasore $(5,15,40 \mu \mathrm{M})$ for 1, 2 and 3 days. DMSO was used as the vehicle control. Cells were harvested each day and assayed for ALP activity and then normalized for total protein. Asterisks indicate significance $(\mathrm{p}<0.05)$ as determined by student $\mathrm{t}$-Test relative to controls for each day. All experiments were reproduced 3-4 times. (D) Calvarial osteoblasts were treated with dynasore $(10 \mu \mathrm{M})$ or DMSO vehicle for 24 hours. RNA was extracted and used for QPCR analysis of osteoblast markers (c-fos, osterix, osteocalcin and type I collagen). QPCR was performed in duplicate and the results from 3 separate experiments were averaged. Statistical significance $(\mathrm{p}<0.05)$ is indicated by the asterisk $(*)$. 
A.

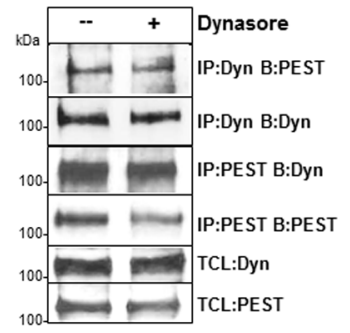

C.
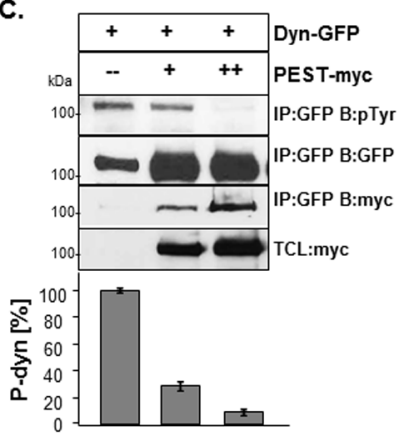

E.

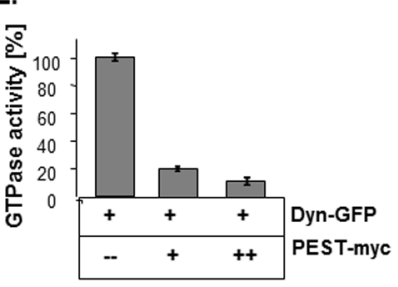

B.

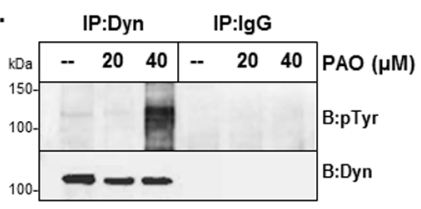

D.

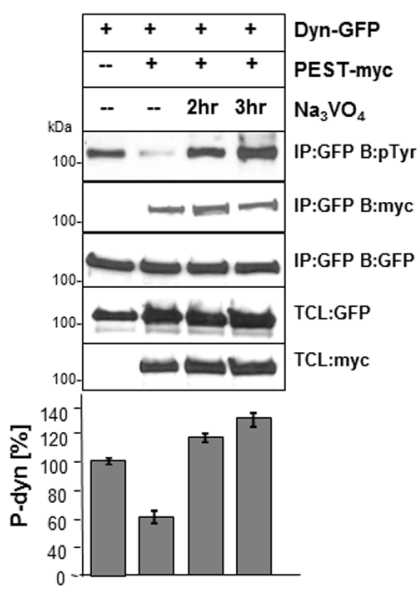

F.

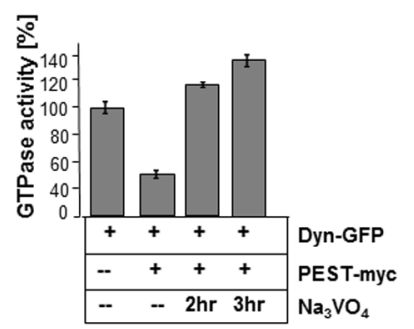

Fig. 3. Dynamin is dephosphorylated by PTP-PEST

(A) Calvarial osteoblasts were treated with dynasore $(40 \mu \mathrm{M})$ or vehicle for $1 \mathrm{hr}$.

Endogenous dynamin and PTP-PEST (PEST) were immunoprecipitated (IP) and blotted as indicated. (B) Calvarial osteoblasts were treated with PAO $(20$ and $40 \mu \mathrm{M})$ or vehicle control for $1 \mathrm{hr}$. Lysates were subject to IP and Western blotting with an antibody to phospho-tyrosine (pTyr) residues. Blots were striped and re-blotted for dynamin to confirm equal amount of dynamin in each IP. IPs were also performed with an IgG antibody. Total cell lysates (TCL). (C) Dynamin (dynWT-GFP) was co-expressed with increasing amounts of PTP-PEST (myc-tagged) in 293VnR cells. Dynamin was immunoprecipitated with antiGFP and blotted with anti-pTyr. PTP-PEST was detected with an antibody to myc. To determine changes in dynamin phosphorylation, densitometric analysis of dynamin pTyr blots was performed and results were normalized for total dynamin without PTP-PEST. Results are expressed as \% dynWT (bar graph). TCLs were blotted with GFP or myc to detect expression of transfected proteins. (D) DynWT and PTP-PEST were expressed as above and cells were treated with $2 \mathrm{mM} \mathrm{Na}_{3} \mathrm{VO}_{4}$ for 2 and $3 \mathrm{hrs}$. Dynamin phosphorylation was analyzed by IP and Western blotting using a pTyr antibody, followed by densitometry. Results are expressed as a \% of dynWT. (E) Lysates from cells expressing dynWT and PTPPEST (from 4C) were subject to IP and assayed using the GTPase assay. Results are expressed as a \% of dynWT. The expression of dynWT and PTP-PEST was confirmed by Western blotting (see 4C). (F) 293VnR cells expressing dynWT and PTP-PEST that were treated with $\mathrm{Na}_{3} \mathrm{VO}_{4}$ (same lysates as in 4D) were assayed for GTPase activity. All samples were prepared in duplicate and results were averaged and expressed as a percentage of dynWT (no PTP-PEST). Similar findings were observed when cells were treated with PAO (data not shown). Labels: IP, immunoprecipitation; B, Western blot; TCL, total cell lysates. 
Experiments were replicated at least 3-5 times with similar findings and representative experiments are shown. 
A.

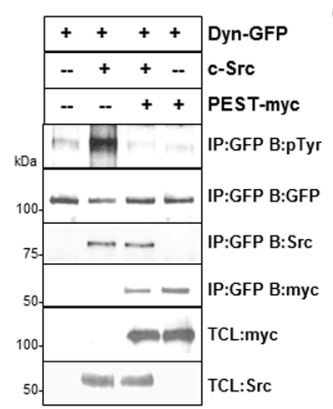

c.
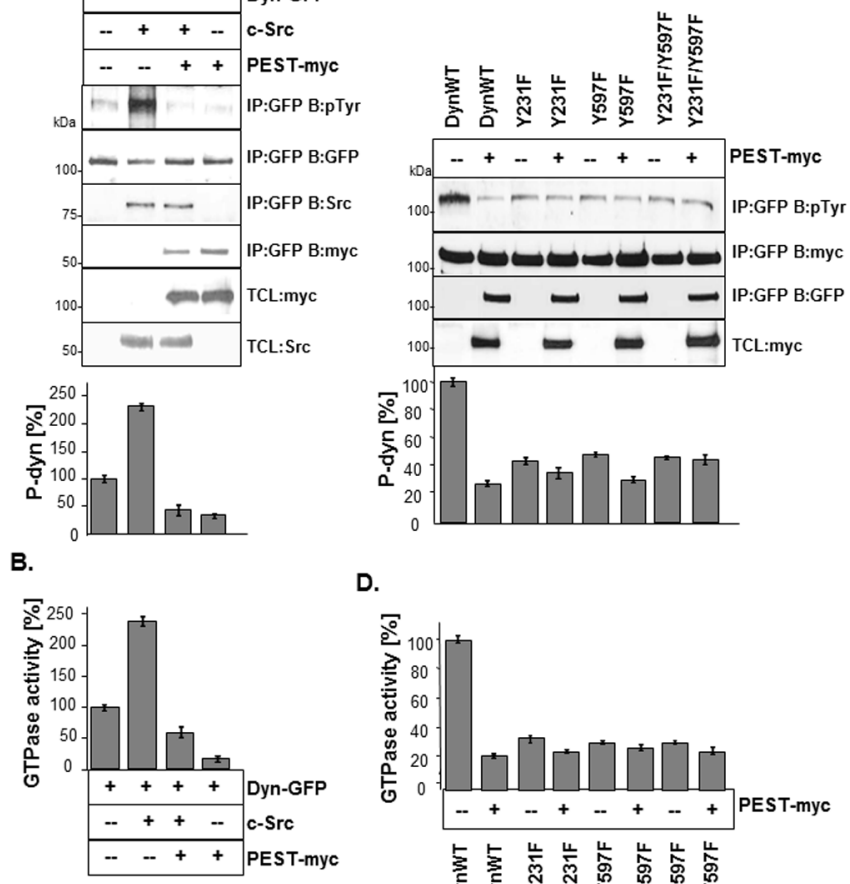

D.

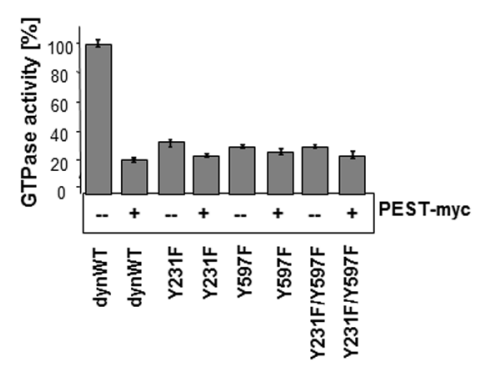

Fig. 4. Src stimulation of dynamin phosphorylation and activity is reversed by PTP-PEST (A) Dynamin and Src were co-expressed with or without PTP-PEST in 293VnR cells. Dynamin was subject to IP and blotted as indicated. Densitometric analysis of phosphorylated dynamin was performed and results were expressed as a percentage relative to dynWT. Findings reveal $140 \%$ increase in dynamin phosphorylation by Src and $192 \%$ decrease in phosphorylation when PTP-PEST was co-expressed with dynWT and Src. (B) The GTPase activity of dynamin in cell lysates (prepared as in 6A) was assayed and expressed as $\%$ of dynWT control. Samples were prepared in duplicate and results were averaged and expressed as \% of dynWT. Experiments were replicated at least 3 times and representative experiments are shown. (C) DynWT, dynY231F, dynY597F and dynY231F/ Y597F were expressed in $293 \mathrm{VnR}$ cells in the presence or absence of PTP-PEST. Dynamin was subject to IP and Western blotting as indicated. Densitometric analysis of phosphorylated dynamin was performed and results were expressed as \% relative to dynWT. (D) Lysates were assayed for dynamin GTPase activity and results were expressed as a \% relative to dynWT alone. 

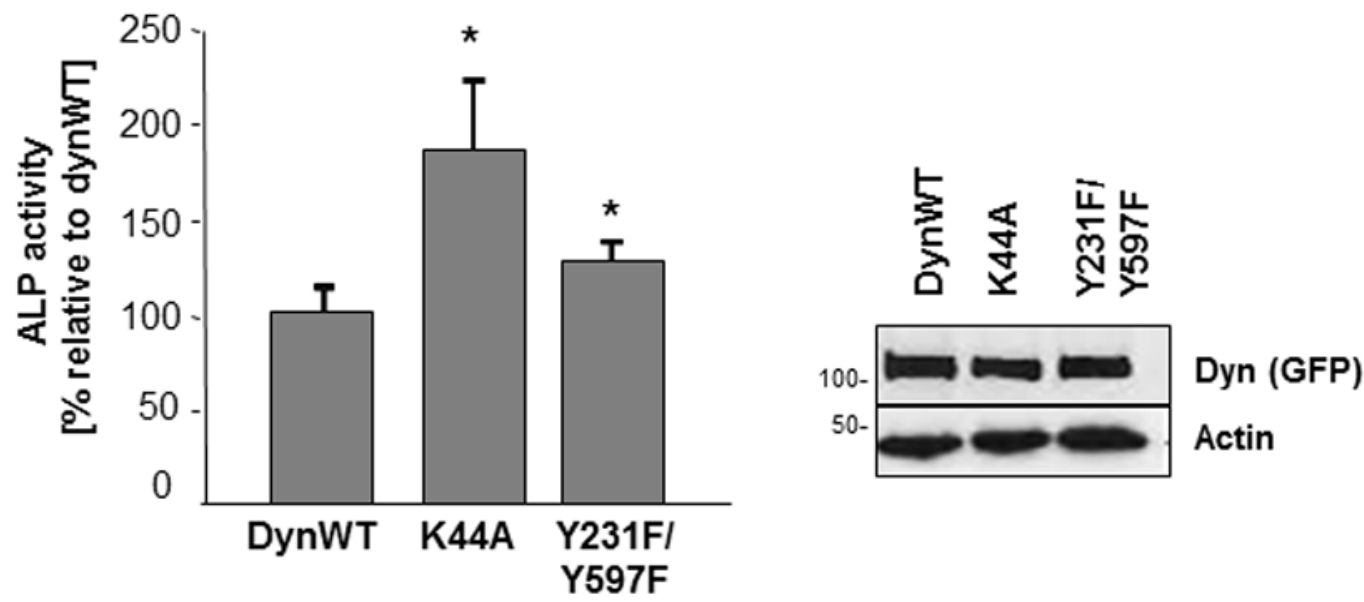

Fig. 5. DynK44A and dynY231F/Y597F affect ALP activity

Equal amounts of dynWT, dynK44A and dynY231F/Y597F were expressed in MC3T3-E1 cells. After 3 days in osteogenic media, cells were harvested and lysates were assayed for ALP activity. Results were normalized for total protein. Western blotting of TCL with an anti-GFP antibody was used to confirm equal level of expression of transfected dynamin. Actin was used as a loading control. Experiments were performed in triplicate and the average \pm SEM is shown. Statistical significance $(\mathrm{p}<0.05)$ compared to dynWT is indicated by the asterisk. 
A.

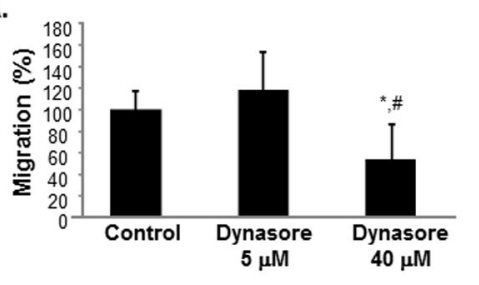

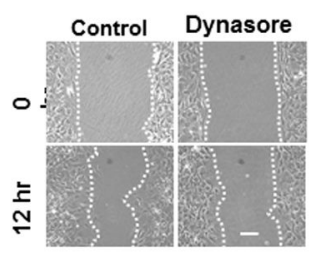

B.

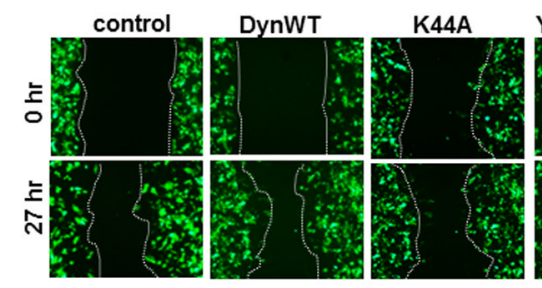

Y231F/Y597F
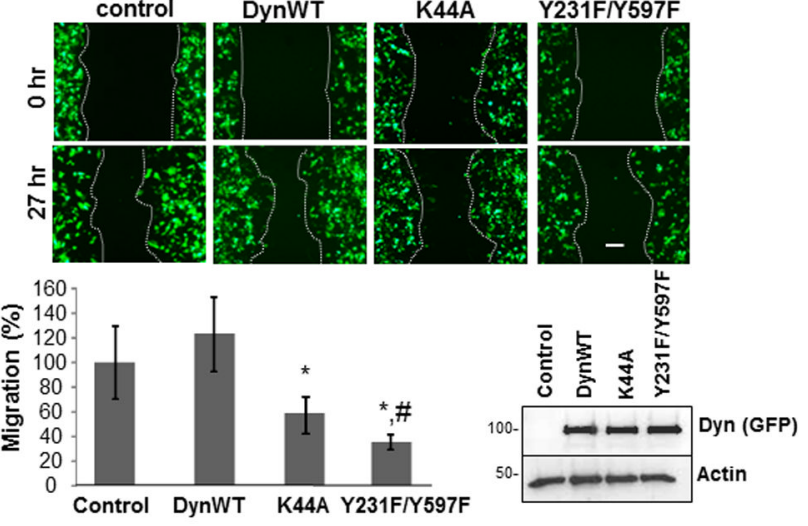

c.

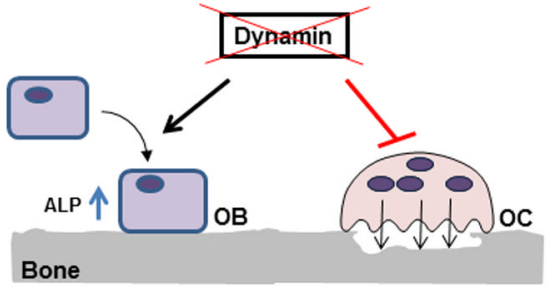

Fig. 6. Dynamin activity and phosphorylation regulate osteoblast migration

(A) Calvarial osteoblasts were seeded the Ibidi $\mu$-Dish migration chambers overnight. After removing the culture insert, cells were incubated with and without dynasore $(5$ or $40 \mu \mathrm{M})$. Cells were imaged immediately $(0 \mathrm{hr})$ and at 12 hours. The distance traveled was quantified by microscopy and results are expressed as a \% of cells control. Representative images are shown and the dashed line indicates the edge of migrating cells. Experiments were performed 3-4 times, each in duplicate, and the average \pm SEM is shown. Statistical analysis was performed using Student's t-Test. Significance for the $40 \mu \mathrm{M}$ dynasore group compared to the control group or $5 \mu \mathrm{M}$ group is indicted by (*) and (\#), respectively. (B) MC3T3-E1 cells expressing empty GFP vector, dynWT-GFP, dynK44A-GFP, or dynY231F/Y597FGFP were replated onto glass coverslips. A cell scraper was used to remove cells from the center of the well. Cells were imaged for GFP at $0 \mathrm{hr}$ and $27 \mathrm{hrs}$. Migration was quantified by microscopy and expressed as a \% relative to GFP-expressing cells. Western blot analysis confirmed equal expression of dynWT, dynK44A and dynY231F/Y597F. Experiments were performed in triplicate and repeated 3 times with similar results. The result of a representative experiment is shown. Statistical significance for dynK44A versus control (*), dynY213F/Y597F versus control (*) and dynY213F/Y597F versus dynK44A (\#) is indicated. Scale bar indicates $10 \mu \mathrm{m}$. (D) Schematic summary of our findings. Our current studies demonstrate that endogenous dynamin plays a role in osteoblast function and that inhibition of dynamin activity increases ALP activity and decreases osteoblast migration. We also reported that inhibition of dynamin GTPase activity decreased the bone resorbing 
activity and the migration of osteoclasts in vitro (Bruzzaniti et al., 2005) (OB, osteoblast; OC, osteoclast). 